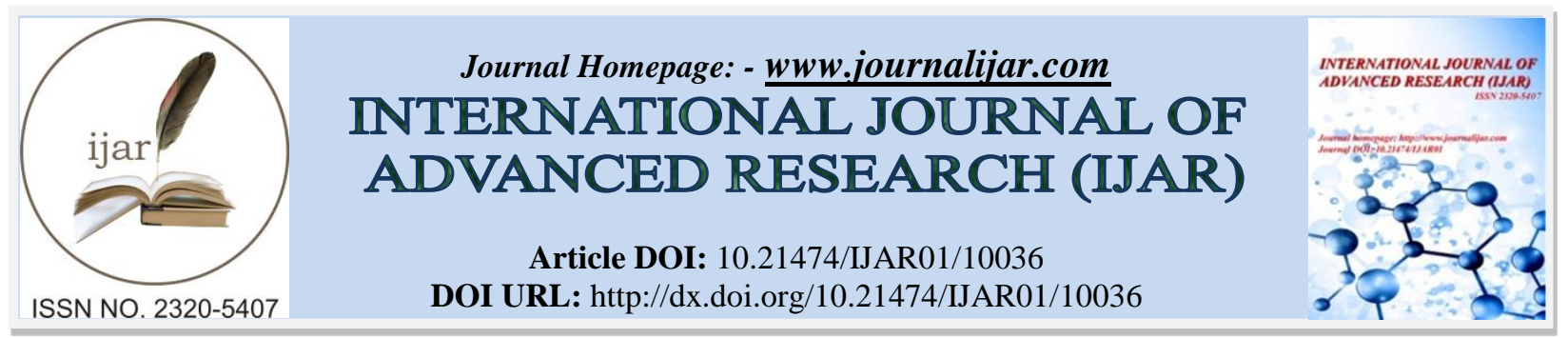

RESEARCH ARTICLE

\title{
AMYLOSE RENALE COMPLIQUANT UN LUPUS ERYTHEMATEUX DISSEMINE RENAL AMYLOIDOSIS IN SYSTEMIC LUPUS ERYTHEMATOSUS.
}

Charifa bassit, MounaZahlane, Leila Benjilali and LamiaaEssaadouni.

Service de médecine interne CHU Mohamed VI ,université caddi ayad Marrakech ,Maroc.

\section{Manuscript Info}

\section{Manuscript History}

Received: 10 September 2019

Final Accepted: 12 October 2019

Published: November 2019

Key words:-

amyloidosis, Lupus, , proteinuria,

biopsy, inflammatory.

\begin{abstract}
Kidney amyloidosis is mainly seen in chronic inflammatory and infectious diseases, rheumatoid arthritis, ankylosing spondylitis and auto-immune diseases. It is exceptional during systemic lupus erythematosus. We report the case of a patient with systemic lupus erythematosus associated with the early onset of kidney amyloidosis type AA .The kidney biopsy shows amyloidosis type AA. This association has been reported in the literature in twenty cases previously. This case study emphasizes the importance of considering the diagnosis of amyloidosis when the findings include massive proteinuria in all stages of systemic lupus erythematosus.
\end{abstract}

Copy Right, IJAR, 2019,. All rights reserved.

\section{Introduction:-}

Le lupus érythémateux systémique (LES) est une maladie auto-immune dotée d'un grand polymorphisme clinique. L'amylose est un vaste groupe de maladies diagnostiquées histologiquement par la présence de dépôts protéiques insolubles dans les tissus [2]. Elle se voit essentiellement au cours des maladies inflammatoires et infectieuses chroniques (polyarthrite rhumatoïde (PR), spondylarthrite ankylosante (SPA), maladies auto-inflammatoires, dilatation des bronches, tuberculose ou de rares processus tumoraux. D'autres facteurs notamment génétiques sont impliqués dans la susceptibilité de survenue de 'amylose AA.

La survenue d'une amylose au cours du lupus est rare.On dénombre environ une vingtaine de cas dans la littérature, nous en rapportons une nouvelle observation.

\section{Observation:-}

Mme H.E âgée de 62 ans ,sans antécédents pathologiques particuliérs,suivie dans notre formation depuis 2014 pour LES à déclaration tardive avec atteinte cutanée(érythéme malaire,photosensibilité), articulaire(polyarthralgies inflammatoire),immunologique(anticorps anti-nucléaire positive, anticorps anti DNA positive),vasculaire(épisode d'embolie pulmonaire massive), rénale(insuffisance rénale avec créatininémie à $16 \mathrm{mg} / \mathrm{l}$ (DFG à $35 \mathrm{ml} / \mathrm{mn}$ ), l'étude histologique a objectivé des lésions glomérulaires minimes.

La patiente a reçu $12 \mathrm{~g}$ de cyclophosphamide devant l'atteinte vasculaire grave.

En Mars 2017 la patiente a été hospitalisée pour aggravation de la fonction rénale avec des infections urinaires à répétition.

Corresponding Author:- Charifa bassit.

Address:-Service de médecine interne CHU Mohamed VI ,université caddi ayad Marrakech ,Maroc. 
Une deuxième ponction biopsie rénale a été réalisé , qui a objectivé une amylose rénale type AA. Ce geste a été indiqué devant des chiffres élevés de protéinurie et dégradation de la fonction rénale(DFG à $5 \mathrm{ml} / \mathrm{mn}$ ).

La patiente est décédée quatre mois après le diagnostic d'amylose rénale.

\section{Discussion:-}

L'amylose est un terme générique qui fait référence à un groupe de maladies secondaires aux dépôts, dans les tissus extracellulaires, de fibrilles. Aujourd'hui, au moins 23 protéines précurseur de fibrilles amyloïdes ont été identifiées. La survenue d'une amylose rénale est considérée actuellement comme exceptionnelle au cours de l'évolution d'un lupus érythémateux disséminé (LED). A notre connaissance, moins de 20 cas ont été reportés dans la littérature anglaise (Tableau I).

Le délai entre le diagnostic du LED et l'installation des symptômes de l'amylose AA varie entre 1 et 35 ans. Dans à peu près la moitié des observations, l'amylose AA apparait dans les cinq ans qui suivent le début du LED. Les concentrations du SAA dans le LED et dans les maladies inflammatoires chroniques ont été étudiées par De Beer et al [10].Ils retrouvèrent des concentrations très élevées de SAA chez les patients présentant une polyarthrite rhumatoïde, une arthrite juvénile chronique et une maladie de Crohn. Elles étaient fortement corrélées à l'activité de la maladie. Contrairement aux concentrations dosées dans la RCH et chez la majorité des patients présentant le LED, qui n'étaient que légèrement élevées même quand la maladie était très active. Les concentrations de la CRP chez les patients avec un LED changent suivant le même model, que celles du SAA.

Les concentrations de la CRP, de façon similaire à celles du SAA, sont souvent normales même en présence de maladie active. Ces différences de concentrations du SAA et de la CRP respectivement entre le LED et la polyarthrite rhumatoïde peuvent être la raison majeur derrière la différence de l'incidence de l'amylose AA entre ces deux pathologies.

Garcia-Tobaruela et al [11] a observé une amélioration des symptômes lors de la mise en place d'un traitement à base de colchicine, mais notre patiente ayant reçu un traitement de colchicine sans bénéfice notable.

Le traitement de l'amylose est peu codifié, son évolution étant directement liée à la maîtrise de l'inflammation et de la concentration de la protéine SAA. Les inhibiteurs de l'interleukine 1 et du TNF semblent avoir de bons résultats sans réelle supériorité prouvée aux immunosuppresseurs type cyclophosphamide ou chlorambucil.

\section{Conclusion:-}

L'apparition d'une amylose secondaire au cours des rhumatismes inflammatoires chroniques comme la polyarthrite rhumatoïde, l'arthrite chronique juvénile et les spondylarthropathies est bien connue. Elle est exceptionnelle au cours du lupus systémique. Elle survient chez des patients ayant un lupus évoluant depuis plusieurs années avec des délais diagnostiques variables.

\section{Conflits d'intérêts:}

Les auteurs déclarent n'avoir aucun conflit d'intérêt.

\begin{tabular}{|l|l|l|l|l|l|}
\hline & Auteur & Sexe/Age & $\begin{array}{l}\text { Type de } \\
\text { biopsie }\end{array}$ & $\begin{array}{l}\text { Délai lupus / } \\
\text { amylose } \\
\text { ans })\end{array}$ & Évolution \\
\hline Cas 1 & $\begin{array}{l}\text { O. Wegelius(1956) } \\
{[1]}\end{array}$ & F/26 & PBR & $\begin{array}{l}\text { LED depuis } \\
5\end{array}$ & Décès de la patiente \\
\hline Cas 2 & $\begin{array}{l}\text { L. A. H/65 } \\
\text { Schleissner(1976) } \\
{[2]}\end{array}$ & PBR & $\begin{array}{l}\text { LED depuis } \\
10\end{array}$ & Décès de la patiente \\
\hline Cas 3 & $\begin{array}{l}\text { S. Webb(Mai 1979) } \\
{[3]}\end{array}$ & F/62 & PBR & $\begin{array}{l}\text { LED depuis } \\
25\end{array}$ & Décès de la patiente \\
\hline Cas 4 & $\begin{array}{l}\text { D. Huston(1981) } \\
{[4]}\end{array}$ & H/43 & PBR & $\begin{array}{l}\text { LED depuis } \\
20\end{array}$ & La protéinurie est stable \\
\hline Cas 5 & RA. & H/59 & PBR & LED depuis & Diminution de la protéinurie \\
\hline
\end{tabular}




\begin{tabular}{|l|l|l|l|l|l|}
\hline & $\begin{array}{l}\text { Bear(Septembre } \\
\text { 1981) [5] }\end{array}$ & & & 3 & \\
\hline Cas 6 & $\begin{array}{l}\text { G. Martin (1984) } \\
\text { [6] H/56 }\end{array}$ & PBR & $\begin{array}{l}\text { LED depuis } \\
10\end{array}$ & Décès du patient \\
\hline Cas 7 & $\begin{array}{l}\text { T. Bestsuyaku Mai } \\
\text { 1993) [7] }\end{array}$ & F/57 & PBR & $\begin{array}{l}\text { LED depuis } \\
5\end{array}$ & $\begin{array}{l}\text { Aggravation de la fonction } \\
\text { rénale }\end{array}$ \\
\hline Cas 8 & $\begin{array}{l}\text { G. Queffeulou } \\
\text { (1998) [8] }\end{array}$ & F/35 & PBR & $\begin{array}{l}\text { LED depuis } \\
4\end{array}$ & $\begin{array}{l}\text { Aggravation de la fonction } \\
\text { rénale }\end{array}$ \\
\hline Cas 9 & $\begin{array}{l}\text { A. García- } \\
\text { Tobaruela (1995) } \\
{[9]}\end{array}$ & H/37 & $\begin{array}{l}\text { Biopsie } \\
\text { hépatique }\end{array}$ & $\begin{array}{l}\text { LED depuis } \\
19\end{array}$ & Stabilisation de la maladie \\
\hline Cas 10 & Notre patiente 2017 & F/62 & PBR & $\begin{array}{l}\text { LED depuis } \\
\text { 03 ans }\end{array}$ & Décès de la patiente \\
\hline
\end{tabular}

Tableau I:-Synthése des cas d'association amylose-lupus.

\section{Bibliographie:-}

1. Wegelius O. Amyloidosis of the kidneys, adrenals and spleen as a complication of acute disseminated lupus erythematosus treated with ACTH and cortisone. Acta Med Scand 1956; 156: 91-95.

2. Schleissner LA, Sheehan WW, Orselli RC. Lupus erythematosus in a patient with amyloidosis, adrenal insufficiency and subsequent immunoblastic sarcoma. Arthritis Rheum 1976; 19: 249-254.

3. Webb S,Segura F, Cervantes F et al. Systemic lupus erythematosus and amyloidosis. ArthritisRheum 1979; 22: 554-556.

4. Huston D, McAdam K P W, Balow J E, Bass R. Amyloidosis in systemic lupus erythematosus. Am J Med 1981; 70: 320- 323.

5. Bear RA, Lang AP. Amyloidosis and systemic lupus erythematosus. Hum Pathol 1981; 12: 853-856 6- Martin G. Ridley, Peter J. Maddison, Colin R. Tribe.Amyloidosis and systemic lupus erythematosus.Annals of the Rheumatic Diseases 1984; 43: 649-650

6. Betsuyaku T,Adachi $\mathrm{T}$, Haneda $\mathrm{H}$ et al. A secondary amyloidosis associated with systemic lupus erythematosus. Intern Med 1993; 32: 391-396.

7. Guillaume Q, Francois B, Catherine M, Béatrice M, Françoise M. AA amyloidosis in systemic lupus erythematosus: an unusual complication. Nephrol Dial Transplant 1998; 13: 1846-1848.

8. Garcia-Tobaruela A, Gil A, Lavilla P, Larrauri J, Pizarro A, Moreno de la Santa C, Lopez-Dupla M, Martinez P. Hepatic

9. Beer.F, Fagan.E, Hughes.G, Mallya.R, Lanham.J, Pepys.M. Serum amyloid A protein concentration in inflammatory diseases and its relationship to the incidence of reactive systemic amyloidosis. Lancet 1982; 31: 231-3.

10. Garcia.A, Gil.A, Lavilla.P . Hepatic amyloidosis associated with systemic lupus erythematosus. Lupus 1995; 4 : 75-77. 